\title{
Baseball Catching Patterns Differ According to Task Constraints
}

\author{
Daiki Murase1, Keiko Yokoyama², Keisuke Fujii',3, Yumiko Hasegawa4, Yuji Yamamoto² \\ ${ }^{1}$ Deajon Junggu Little Baseball Club, Deajon, Korea \\ ${ }^{2}$ Research Center of Health, Physical Fitness, and Sports, Nagoya University, Nagoya, Japan \\ ${ }^{3}$ Research Fellowship Division, Japan Society for the Promotion of Science, Tokyo, Japan \\ ${ }^{4}$ Faculty of Humanities \& Social Sciences, Iwate University, Morioka, Japan \\ Email: yamamoto@htc.nagoya-u.ac.jp
}

Received 15 April 2016; accepted 1 August 2016; published 4 August 2016

Copyright (C) 2016 by authors and Scientific Research Publishing Inc.

This work is licensed under the Creative Commons Attribution International License (CC BY).

http://creativecommons.org/licenses/by/4.0/

(c) (i) Open Access

\begin{abstract}
We investigated the effect of task constraints on movement patterns and sought to confirm the finding that the combined movement involved in the transition between catching and throwing emerges through self-organization. We conducted two experiments. In Experiment 1, four participants were required to perform two tasks: catch a launched baseball (catching task) and catch the launched baseball and immediately throw it to a target (catching and throwing task). The balls were launched from five spatial positions, and the participants were instructed to catch the balls with their left hand using a baseball glove. We found that the catching movement differed according to the task and spatial constraints. When the ball was launched close to the body in the catching and throwing task, the shoulder and hip segment angles rotated in the direction of the throw, which resulted in combining the terminal phase of catching with the preparatory phase of throwing. In Experiment 2, two participants were asked to complete the catching and throwing task using the same procedure as in Experiment 1, to investigate the sequence effect. Our findings showed that the spatial position at which the trunk rotation switched direction, that is, hysteresis, differed according to the sequence of positions, suggesting that the combination of two movement patterns, such as catching and throwing, emerged through self-organization.
\end{abstract}

\section{Keywords}

Task Constraint, Catching, Hysteresis

\section{Introduction}

Human movements vary according to organismic, environmental, and task constraints (Newell, 1986). For in-

How to cite this paper: Murase, D., Yokoyama, K., Fujii, K., Hasegawa, Y., \& Yamamoto, Y. (2016). Baseball Catching Patterns Differ According to Task Constraints. Advances in Physical Education, 6, 151-157.

http://dx.doi.org/10.4236/ape.2016.63017 
stance, it takes longer to grasp a disk to place it in a well than to grasp the same disk to throw it into a large box (Marteniuk, MacKenzie, Jeannerod, Athenes, \& Dugas, 1987). This phenomenon is an example of a task constraint, which may influence human movement. In this example, the temporal difference is not the result of combining the grasping and throwing movements; rather, it emerges through self-organization based on the task goals. We investigated the various movement patterns corresponding to task constraints during a sporting activity.

In a number of sports, each movement can be divided into three phases: preparatory, main, and terminal (Meinel, 1960). In tennis, for example, the back-swing, forward-swing, and follow-through strokes correspond to the preparatory, main, and terminal phases, respectively. The consecutive execution of two different movements in succession involves transitioning from the terminal phase of the current movement into the preparatory phase of the next movement, which is the result of self-organization (Suzuki \& Yamamoto, 2015; Yamamoto \& Gohara, 2000). Although previous studies have investigated the patterns of two consecutive movements, we examined the effect of task constraints on successive movements. In other words, we were interested in whether the task constraints would influence the transition from the current movement into the next movement. To this end, we assessed two tasks: catching a baseball and catching and throwing a baseball.

Furthermore, we examined the effect of spatial constraints on combined movement in the catching-throwing task. A previous study showed that when subjects were instructed to grasp a stationary bar and move it from one position to another, they grasped the bar based on the comfort of the final hand and arm positions (Kelso, Buchanan, \& Murata, 1994; Rosenbaum \& Jorgensen, 1992). Switching between overhand and underhand grips is influenced by spatial constraints and has been shown to have a sequence effect (Remington, 1969; Soetens, 1998) and hysteresis in relation to multifunctionality (Kelso, Buchanan, \& Murata, 1994). Hysteresis is a feature of self-organization in which bifurcation points differ depending on the direction of changes in control parameters such as sequence effects. In an example of semantic ambiguity, the transition from the face of a man to a girl is observed at different points depending on the direction of view (Ditzinger \& Haken, 1989; Haken, 2006). Such hysteresis can be observed in human movement. Switching between a forehand and backhand drive in table tennis occurs at different spatial positions, depending on the sequence order; that is, from forehand to backhand or from backhand to forehand (Sørensen, Ingvaldsen, \& Whiting, 2001).

We investigated the catching movement under two task constraints: a catching task alone and a catching and throwing task. Furthermore, we examined the combined movement of the terminal phase of catching and the preparatory phase of throwing in the catching and throwing task. Finally, we examined the effect of the sequence of various spatial positions on hysteresis in the direction of the trunk rotation. Our aims were to identify the different movement patterns associated with the task constraints and to confirm that the combined movement from catching to throwing emerges through self-organization.

\section{Experiment 1}

This experiment aimed to clarify whether differences in movement patterns depend on the task constraints.

\subsection{Method}

\subsubsection{Participants}

The participants were four males who each had more than six years experience playing baseball. They had many experiences like an experimental task as an infielder, so they were familiar with this task. All participants were righthanded and their mean age ( \pm standard deviation) was $23.8 \pm 3.03$. They participated in this experiment in October, 2015. The study protocol was approved by the Internal Review Board of the Research Center of Health, Fitness, and Sports at Nagoya University and conformed to the principles expressed in the Declaration of Helsinki.

\subsubsection{Task and Apparatus}

The participants were required to perform two tasks: catch a launched ball (catching task) and catch the launched ball and throw it to a target (catching and throwing task). In both tasks, participants were asked to stand in a relaxed position with their feet parallel, arms beside their body, and their head upright gazing straight ahead. A hand-made ball-pitching machine was placed $2.7 \mathrm{~m}$ from the participants' frontal plane. After a signal from the experimenter, baseballs were launched from five positions: $0,0.3,0.6,0.9$, and $1.2 \mathrm{~m}$ ranging from the center of the body to the right side of the participants (Figure 1).

The initial height of the ball machine and launch angle were adjusted so that the balls reached the participants 


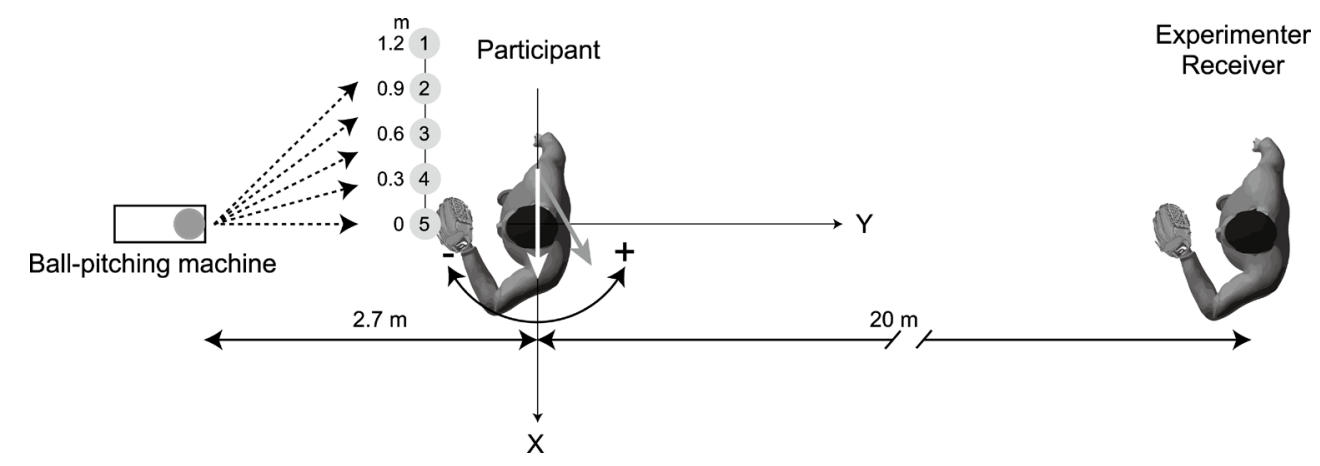

Figure 1. Experimental setup.

at a height of $1.2 \mathrm{~m}$. The accuracy of the ball projection was tested by projecting 240 balls in a previous pilot study. The standard deviation of the projection from a distance of $2.7 \mathrm{~m}$ was $0.026 \mathrm{~m}$ in the horizontal and 0.049 $\mathrm{m}$ in the vertical direction.

In the catching task, the participants were instructed to catch the launched ball with their left hand using a baseball glove. In the catching and throwing task, the participants were instructed to catch the launched ball with their left hand using a baseball glove then immediately throw the ball as accurately as possible to an experimenter with a baseball glove standing $20 \mathrm{~m}$ behind them. The five spatial positions were randomized for all participants in both tasks.

Six optical motion capture cameras (OptiTrack Prime 17W, Natural Point, Inc., Corvallis, OR, USA), operated at $200 \mathrm{~Hz}$, were used to record the participants' movements. A right-handed orthogonal reference frame was used, the Z-axis was vertical, the $\mathrm{X}$-axis was parallel to the vector from the right to the left shoulder, and $\mathrm{Y}$ was perpendicular to the other two axes and pointed at the target. We attached $30 \mathrm{~mm}$ markers to the right and left acromions and posterior inferior iliac spine of the participants to observe trunk rotation, and a $20 \mathrm{~mm}$ marker was attached to the participants' right carpus to measure the timing of the ball release. The baseballs were covered with reflective sheets to be tracked as reflective markers.

\subsubsection{Procedure}

All participants provided written informed consent after the purpose of the study and procedures were explained. After a sufficient warm-up period, the markers were attached and the participants were asked to catch a total of 100 balls launched from five different positions in each task. In both tasks, balls were launched in 10 blocks of 5 trials, for each position. The order of the tasks was randomly assigned across participants. Participants were instructed to stand on a footprint marked on the floor before each trial.

\subsubsection{Analysis}

The shoulder and hip segment angles were defined as the angle between the $X$ vector and the vector from the right to the left shoulder and hip launched onto the X-Y plane, respectively. The zero angle was defined as the neutral orientation of the ball pitching machine (i.e., directly facing the participant), and the clockwise and counterclockwise rotations were defined as negative and positive, respectively. The onset of the ball launch was defined as the peak of the resultant acceleration after the launch. The onset of catching was defined as the point at which the resultant acceleration of a launched ball exceeded $\pm 100 \mathrm{~ms}^{-2}$. The onset of ball release was defined as the point at which the distance between the right carpus and the ball exceeded the measured length between the right carpus and the tip of the right middle finger. Two-way repeated measures analyses of variance (ANOVAs) (Task: $2 \times$ Catch position: 5) were used to test differences in the shoulder and the hip angles at the onset of launching and of catching the ball. The effect size was calculated as $\hat{\eta}_{G}^{2}$ (Olejnik \& Algina, 2003). All statistics were calculated by car package and customized program using R programming language.

\subsection{Results}

\subsubsection{Preparatory Phase}

The two-way repeated measures ANOVAs revealed no significant main effects of task and position and no interaction effect for shoulder and the hip angle at the onset of the ball launch, indicating that the shoulder and hip 
angles did not differ according to task or spatial constraints at the onset of the ball launch (Figure 2(a) and Figure 2(b)).

\subsubsection{Catching Phase}

The analysis of the shoulder angle at the onset of catching the ball revealed a significant interaction effect between task and position $\left(\mathrm{F}=3.60, \mathrm{df}=4,12, p<0.05, \hat{\eta}_{\mathrm{G}}^{2}=0.0395\right)$. Analysis of the simple main effect revealed that the shoulder angle was greater in the catching and throwing task than in the catching task at positions $4(0.3 \mathrm{~m})$ and $5\left(0 \mathrm{~m}\right.$; position 4: $\mathrm{F}=7.23, \mathrm{df}=1,5, p<0.05, \quad \hat{\eta}_{\mathrm{G}}^{2}=0.3274$, position 5: $\mathrm{F}=10.79, \mathrm{df}=1,5, p<$ $0.05, \hat{\eta}_{\mathrm{G}}^{2}=0.4207$ ). The simple main effect of position was significant for both tasks (catching task: $\mathrm{F}=9.22$, $\mathrm{df}=4,14, p<0.001, \hat{\eta}_{\mathrm{G}}^{2}=0.6058$; catching and throwing task: $\mathrm{F}=16.54, \mathrm{df}=4,14, p<0.001, \hat{\eta}_{\mathrm{G}}^{2}=$ 0.7338). Tukey's honest significant difference (HSD) post-hoc analysis revealed that in the catching task, the shoulder angle at catching position $1(1.2 \mathrm{~m})$ was significantly smaller than those at positions $4(\mathrm{q}=5.988, \mathrm{df}=$ $14, p<0.01)$ and position $5(\mathrm{q}=7.708, \mathrm{df}=14, p<0.001)$. The shoulder angle at position $2(1.0 \mathrm{~m})$ was significantly smaller than that at position $5(\mathrm{q}=5.443, \mathrm{df}=14, p<0.05)$. Furthermore, the post-hoc analysis revealed that in the catching and throwing task, the shoulder angle at position 1 was significantly smaller than those at positions $3(0.9 \mathrm{~m})(\mathrm{q}=5.827, \mathrm{df}=14, p<0.01), 4(\mathrm{q}=8.113, \mathrm{df}=14, p<0.001)$, and $5(\mathrm{q}=10.451, \mathrm{df}=14, p$ $<0.001)$, and the angle at position 2 was smaller than those at positions $4(\mathrm{q}=4.726, \mathrm{df}=14, p<0.05)$ and 5 (q $=7.065$, $\mathrm{df}=14, p<0.01)$. The shoulder angle at position 3 was smaller than that at position $5(\mathrm{q}=4.623, \mathrm{df}=$ $14, p<0.05)$.

The two-way ANOVA for hip angle at the onset of catching revealed a significant interaction effect between task and position $\left(\mathrm{F}=7.37, \mathrm{df}=4,12, p<0.001, \hat{\eta}_{\mathrm{G}}^{2}=0.0394\right)$. The simple main effects analysis revealed that the hip angle was greater in the catching and throwing task than in the catching task at position $5(\mathrm{~F}=8.46, \mathrm{df}=$
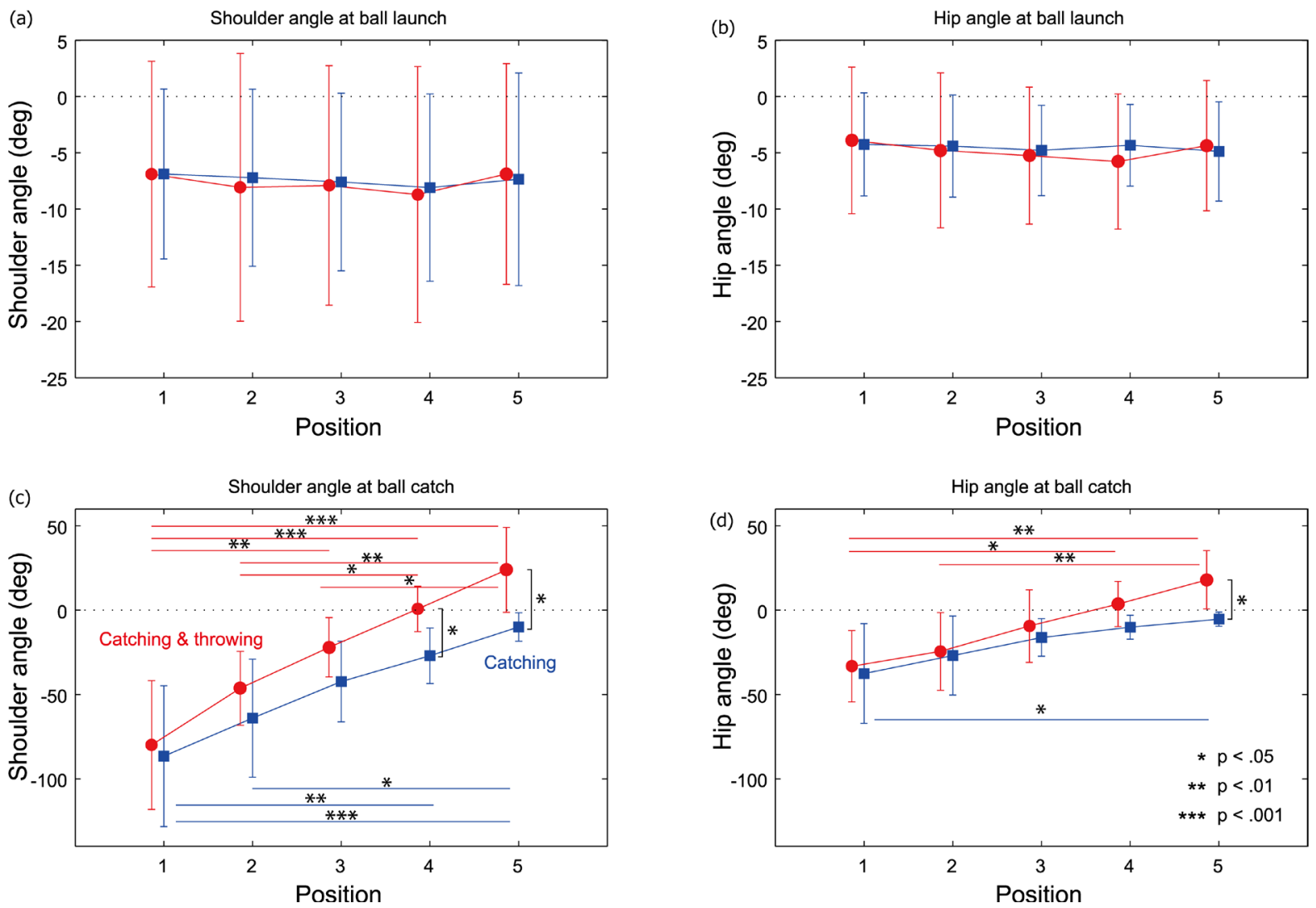

Figure 2. Changes in the shoulder and hip angles in the catching and throwing tasks. Means and standard deviations are shown for the catching (blue squares) and catching and throwing (red circles) task conditions and five spatial conditions. Panels (a) and (b) show the shoulder and hip angles at the onset of the ball launch, respectively. Panels (c) and (d) show the shoulder and hip angles at the onset of catching, respectively. 
1, 4, $p<0.05, \hat{\eta}_{\mathrm{G}}^{2}=0.3607$ ). The simple main effect of position was significant in both tasks (catching task: $\mathrm{F}$ $=3.62, \mathrm{df}=4,13, p<0.001, \hat{\eta}_{\mathrm{G}}^{2}=0.3763$, catching and throwing task: $\mathrm{F}=9.15, \mathrm{df}=4,13, p<0.001, \hat{\eta}_{\mathrm{G}}^{2}=$ 0.6040). Tukey's post-hoc analysis revealed that in the catching task, the hip angle was significantly smaller at position $1(1.2 \mathrm{~m})$ than at position $5(\mathrm{q}=4.702, \mathrm{df}=14, p<0.05)$. In the catching and throwing task, the hip angle was significantly smaller at position 1 than at positions $4(\mathrm{q}=5.397, \mathrm{df}=14, p<0.05)$, and $5(\mathrm{q}=7.488$, $\mathrm{df}$ $=14, p<0.01$ ), and the angle at position 2 was smaller than that at position $5(\mathrm{q}=6.191$, $\mathrm{df}=14, p<0.01)$.

Our findings showed that the shoulder and hip angles differed at the onset of catching a ball when it was launched close to the body of the participant, suggesting that the movement patterns differed depending on spatial and task constraints (Figure 2(c) and Figure 2(d)).

\section{Experiment 2}

Experiment 2 investigated the sequence effect of the launching position on hysteresis in the catching and throwing movement.

\subsection{Method}

\subsubsection{Participants}

Two of the four males who participated in Experiment 1 rotated in a counterclockwise direction in every trial in the catching and throwing task, regardless of the spatial constraint. The two participants who turned in clockwise and counterclockwise directions, depending on the launching position, were included in Experiment 2.They participated in this experiment in October, 2015.

\subsubsection{Task}

The participants were asked to perform a catching and throwing task similar to that in Experiment 1 . The orders of launch position were both ascending and descending series, which differed from Experiment 1. One participant had an ascending series first, while another had a descending series first.

\subsubsection{Procedure}

The procedure was the same as that used in Experiment 1. Baseballs were launched in 10 blocks of 5 trials in which the series of five spatial positions was repeated in an ascending or descending order.

\subsubsection{Analysis}

The definitions of shoulder and hip segment angles were the same as those in Experiment 1. We plotted the angle trajectories and angular velocities of the shoulder and hips in the phase plane to detect differences in the movement patterns. The frequency of clockwise and counterclockwise rotations was calculated for each position.

\subsection{Result}

\section{Hysteresis in the Catching and Throwing Task}

Figure 3 shows the angle trajectories and angular velocities of the hips in the phase plane and the frequency of clockwise and counterclockwise rotations under the ascending and descending conditions. Participant 1 had a clockwise rotation in 1 of 10 trials at position 3 under the descending, but not ascending condition. Participant 2 showed a clockwise rotation in 2 of 10 trials at position 4 under the descending condition and in 1 of 10 trials under the ascending condition. These findings show that in the catching and throwing task, the position at which the direction of rotation was switched was affected by the sequence order.

Our findings suggest that the sequence effect was responsible for the emergence of hysteresis. Accordingly, the combined catching and throwing movement showed multifunctionality, with two stable states consisting of clockwise and counterclockwise trunk rotations. Because the sequence effect influenced the transition point, these movements are considered to be self-organized.

\section{Discussion}

We investigated the movements involved in catching a baseball under two task constraints, catching the ball and catching and throwing the ball, and five spatial constraints. 



Figure 3. Hysteresis during the catching and throwing movements. Panels (a) and (c) show the angle trajectory and angular velocity of the hips in the phase plane under the ascending condition in participants 1 and 2, respectively. Panels (b) and (d) show the angle trajectory and angular velocity of the hips in the phase plane under the descending condition in participants 1 and 2, respectively. Panels (e)-(h) show the frequencies of clockwise (CW) and counterclockwise (CCW) rotations corresponding to (a)-(d).

We found that the catching movement was affected by the task and spatial constraints. When the ball was launched close to the body in the catching and throwing task, the shoulder and hip segment angles rotated in the direction of the throw, in which case, the terminal phase of catching transitioned into the preparatory phase of throwing. In contrast, the shoulder and the hip segment angles did not rotate when the ball was launched during the catching task because it was not necessary to transition to the next movement phase. Taken together, our findings suggest that when an individual performs several movements successively, the terminal phase of the previous movement changes depending on the task goal, which may be considered a task constraint (Marteniuk et al., 1987).

Our findings offer insight for learning sport-related movement patterns, particularly for ball games in which several movement patterns are performed successively, such as in catching and throwing a baseball or touching and kicking a football (Nakayama, 2007). When mastering these skills, it is not sufficient to learn only the catching or touching movement. Our findings suggest that the movement patterns involved in catching or touching a ball differ from those produced when a ball is caught then thrown or touched then kicked. Moreover, when a temporal constraint is severe, it is necessary to transition from the current to the next movement quickly. Thus, learning the combination phase, which involves the terminal phase of the current movement and preparatory phase of the next movement, is important for the development of sporting skills.

We further investigated the combination phase by examining the sequence effect of various spatial positions on hysteresis in the direction of trunk rotation. 
We found that the spatial position at which the trunk rotation switched direction differed according to the sequence of positions. Previous studies have shown that hysteresis caused by the sequence effect provided evidence of self-organization in various phenomena (Ditzinger \& Haken, 1989; Haken, 2006; Hristovski et al., 2006; Sørensen et al., 2001). Thus, our results suggest that the combination of two movement patterns, such as catching and throwing, emerge through self-organization. However, Experiment 2 included only two participants; thus, our results have a limitation for generalization, further studies are needed to confirm our findings.

\section{Acknowledgements}

This work was partially supported by Grants-in-Aid for Scientific Research (A) 24240085 from MEXT, Japan.

\section{References}

Ditzinger, T., \& Haken, H. (1989). Oscillations in the Perception of Ambiguous Patterns. Biological Cybernetics, 61, 279287. http://dx.doi.org/10.1007/BF00203175

Haken, H. (2006). Synergetics of Brain Function. International Journal of Psychophysiology, 60, 110-124. http://dx.doi.org/10.1016/j.ijpsycho.2005.12.006

Hristovski, R., Davids, K., Araújo, D., \& Button, C. (2006). How Boxers Decide to Punch a Target: Emergent Behavior in Nonlinear Dynamical Movement Systems. Journal of Sports Science and Medicine, 5, 60-73.

Kelso, J. A. S., Buchanan, J. J., \& Murata, T. (1994). Mutifunctionality and Switching in the Coordination Dynamics of Reaching and Grasping. Human Movement Science, 13, 63-94. http://dx.doi.org/10.1016/0167-9457(94)90029-9

Marteniuk, R. G., MacKenzie, C. L., Jeannerod, M., Athenes, S., \& Dugas, C. (1987). Constraints on Human Arm Movement Trajectories. Canadian Journal of Psychology, 41, 365-378. http://dx.doi.org/10.1037/h0084157

Meinel, K. (1960). Bewegungslehre. Berlin: Volk und Wissen Volkseigener.

Nakayama, M. (2007). The Effects of Play Area Size as Task Constraints on Soccer Pass Skills. Football Science, 5, 1-6.

Newell, K. M. (1986). Constraints on the Development of Coordination. In M. G. Wade, \& H. T. A. Whiting (Eds.), Motor Development in Children: Aspects of Coordination and Control (pp. 341-360). The Netherlands: Martinus Nijhoff, Dordrecht. http://dx.doi.org/10.1007/978-94-009-4460-2 19

Olejnik, S., \& Algina, J. (2003). Generalized Eta and Omega Squared Statistics: Measures of Effect Size for Some Common Research Designs. Psychological Methods, 8, 434-447. http://dx.doi.org/10.1037/1082-989X.8.4.434

Remington, R. J. (1969). Analysis of Sequential Effects in Choice Reaction Times. Journal of Experimental Psychology, 82, 250-257. http://dx.doi.org/10.1037/h0028122

Rosenbaum, D. A., \& Jorgensen, M. J. (1992). Planning Macroscopic Aspects of Manual Control. Human Movement Science, 11, 61-69. http://dx.doi.org/10.1016/0167-9457(92)90050-L

Soetens, E. (1998). Localizing Sequential Effects in Serial Choice Reaction Time with the Information Reduction Procedure. Journal of Experimental Psychology: Human Perception and Performance, 24, 547-568. http://dx.doi.org/10.1037/0096-1523.24.2.547

Sørensen, V., Ingvaldsen, R. P., \& Whiting, H. T. A. (2001). The Application of Co-Ordination Dynamics to the Analysis of Discrete Movements Using Table-Tennis as a Paradigm Skill. Biological Cybernetics, 85, 27-38. http://dx.doi.org/10.1007/PL00007994

Suzuki, H., \& Yamamoto, Y. (2015). Robustness to Temporal Constraint Explains Expertise in Ball-over-Net Sports. Human Movement Science, 41, 193-206. http://dx.doi.org/10.1016/j.humov.2015.02.009

Yamamoto, Y., \& Gohara, K. (2000). Continuous Hitting Movements Modeled from the Perspective of Dynamical Systems with Temporal Input. Human Movement Science, 19, 341-371. http://dx.doi.org/10.1016/S0167-9457(00)00018-X 


\section{Submit or recommend next manuscript to SCIRP and we will provide best service for you:}

Accepting pre-submission inquiries through Email, Facebook, LinkedIn, Twitter, etc.

A wide selection of journals (inclusive of 9 subjects, more than 200 journals)

Providing 24-hour high-quality service

User-friendly online submission system

Fair and swift peer-review system

Efficient typesetting and proofreading procedure

Display of the result of downloads and visits, as well as the number of cited articles

Maximum dissemination of your research work

Submit your manuscript at: http://papersubmission.scirp.org/ 